\title{
Delay Differential Model for Tumour-Immune System With Chemo-therapy and Optimal Control
}

\author{
Fathalla A. Rihan ${ }^{1, *}$ and Bassel F. Rihan ${ }^{2}$ \\ ${ }^{1}$ Department of Mathematical Sciences, College of Science, Al Ain, 15551, UAE University, UAE \\ ${ }^{2}$ Faculty of Medicine, Ain Shams University, Abbassyia 1156, Cairo, Egypt
}

\begin{abstract}
In this paper, we present a mathematical model of tumour-immune interactions in presence of chemotherapy treatment. The model is governed by a system of delay differential equations with optimal control variables. The control variables are included to justify the best strategy of treatments with minimum side effects, by reducing the production of new tumour cells and keeping the number of normal cells above the average of its carrying capacity. Existence of optimality and optimality conditions are also proved. The numerical simulations show that the optimal treatment strategy reduces the load of tumour cells and increases the effector cells after few days of therapy.
\end{abstract}

Keywords: Chemotherapy; DDEs; Hamiltonian; Immune system; Mathematical modeling; Optimal control; Time-lags

\section{Introduction}

Mathematical modeling of tumour-immune interactions is very complex and has a long history e.g. [1-7]. In [7], Kuznetsov et al. modeled the interactions of cytotoxic T-lymphocyte (CTL) response and the growth of an immunogenic tumour. In other contributions [8-10], the authors take into account the penetration of the tumour cells by the effector cells, which simultaneously causes the inactivation of effector cells. In [11], the authors consider the effects of time-delay on the two-dimensional system which represents the basic model of the immune response. They study variations of the stability of the fixed points due to time-delay and the possibility for the occurrence of the chaotic solutions. Yafia [12] analyzed an interaction between the proliferating and quiescent cells tumour with a single delay. He showed the occurrence of Hopf bifurcation as the delay crosses some critical value [13, 14]. Recently, Rihan et. al [15] proposed a delay differential model with optimal control for tumour-immune system with external treatments.

We also mention here that there are many problems in biosciences (such as epidemics, harvesting, chemostat, treatment of diseases, physiological control, vaccination) which can be addressed within an optimal control framework for systems of DDEs [16-19]. However, the amount of real experience that exists with optimal control problems (OCPs) is still small.

Consider a DDE with an $m$-dimensional control term $u(t)$ of the form

$$
y^{\prime}(t)=f\left(y(t), y\left(t-\tau_{1}\right), y\left(t-\tau_{2}\right), \ldots, y\left(t-\tau_{d}\right), u(t), t\right)
$$

*e-mail: frihan@uaeu.ac.ae 
and a suitable objective functional $\mathcal{J}_{0}(u)$

$$
\operatorname{Minimize} \mathcal{J}_{0}(u)=\int_{0}^{t_{f}} \mathcal{F}\left(y(t), y\left(t-\tau_{1}\right), y\left(t-\tau_{2}\right), \ldots, y\left(t-\tau_{d}\right), u(t), t\right) d t
$$

and subject to control constraint $a \leq u(t) \leq b$, and state constant $y(t) \leq c$, where $a$ and $b$ are the lower and upper bounds. The integrand, $\mathcal{F}(:)$ is called the Lagrangian of objective functional which is continuous in $\left[0, t_{f}\right]$. Additional equality or inequality constraint(s) can be imposed in of $\mathcal{J}_{0}(u)$.

OCPs using differential models were studied in connection with immune responses to infections; See [20]. In [21], delay model with optimal control is used to describe the interactions between HIV, CD4+ T cells, and cell-mediated immune response. Both the treatment and the intracellular delay are incorporated into the model in order to improve therapies to cure HIV infection. The optimal controls represent the efficiency of drug treatment in inhibiting viral production and preventing new infections. A humoral immune response model was considered in the paper [22] on determining optimal intravenous drug delivery in AIDS patients. The objective was to find a control strategy that minimizes the total drug administered subject to the constraint that patient recovers. Recently, the authors in [15], studied an optimal control problem of delay differential model to describe the dynamics of tumour-immune interactions in presence of immuno-chemotherapy. The model includes constant delays in the mitotic phase to justify the time required to stimulate the effector cells and for the effector cells to develop a suitable response to the tumour cells. By applying optimal control theory, the cost associated with the immuno-chemotherapy is minimized to reduce load of of tumour cells.

Herein, we just consider a very simple delayed tumour-immune competition model, without treatments which is presented in [23]. Assume that $E(t)$ represents effector cells population, such as $\mathrm{CD}^{+} \mathrm{T}$ cells and $T(t)$ is the tumour cells population. The model takes the form

$$
\begin{aligned}
& \frac{d E(t)}{d t}=\sigma+\frac{\rho E(t-\tau) T(t-\tau)}{\eta+T(t-\tau)}-\mu E(t-\tau) T(t-\tau)-\delta E(t), \\
& \frac{d T(t)}{d t}=r_{2} T(t)(1-\beta T(t))-n E(t) T(t),
\end{aligned}
$$

where $\sigma$ represents the normal rate (not increased by the presence of the tumour) of the flow of adult effector cells into the tumour side (region). The source of the immune cells is considered to be out side of the system, so it is reasonable to assume a constant influx rate $\sigma . \mu$ is fraction of immune cells inactivated in interactions with tumour cells. In the absence of any tumour, the cells will die at a rate $\delta$. The presence of tumour cells stimulates the immune response, represented by the positive nonlinear growth term for the immune cells $\rho E(t-\tau) T(t-\tau) /(\eta+T(t-\tau))$ where $\rho$ and $\eta$ are positive constants, $\tau \geq 0$ is the timedelay that presents the time needed by the immune system to develop a suitable response after recognizing the tumour cells. The saturation term (Michaelis-Menten form) with the $E(t)$ compartment and logistic term with the $T(t)$ compartment is considered. Of course, the presence of the tumour cells virtually initiates the proliferation of tumour-specific effector cells to reach a saturation level parallel with the increase in the tumour populations. $r_{2}$ is the growth rate of tumour cells. As a result of the interaction, the immune effector cells decrease load tumour cells at rate $n$. 


\section{Delay Differential Model of Tumour-Immune System With Chemotherapy}

To include external chemotherapy in model (3), we should consider extra two variables namely amount of chemotherapy $u(t)$ and normal cells $N(t)$ with control variable $v(t)$. The formulation as an optimal control problem allows us to: (i) investigate the dynamical system of interacting cell populations being affected by the treatments; (ii) Optimize the application of the control such that the quantity of the treatments is optimized; and (iii) Minimize the tumour size at some of end-time; See [15].

We aim to design an efficient treatment protocol, where we employ the tools of optimal control theory. This demonstrates how immunotherapy and chemotherapy might be combined for more effective treatment and to protect the patient from opportunistic infection, as well as fighting the cancer itself. Unlike chemotherapy, immunotherapy does not kill tumour cells directly, but it activates and stimulates the growth of immune cells, most importantly T-Cells, and NK Cells, which are capable of destroying cancer cells directly. Therefore, the main goal of combining immuno-chemotherapy treatment is to eradicate the tumour cells, with the minimum side-effect, while maintaining adequate amounts of healthy tissues. We also assume a homogeneity of the tumour cells. The modified model is

$$
\begin{aligned}
\frac{d E(t)}{d t} & =\sigma+\frac{\rho E(t-\tau) T(t-\tau)}{\eta+T(t-\tau)}-\mu E(t-\tau) T(t-\tau)-\delta E(t)-a_{1}\left(1-e^{-u(t)}\right) E(t), \\
\frac{d T(t)}{d t} & =r_{2} T(t)(1-\beta T(t))-n E(t) T(t)-c_{1} N(t) T(t)-a_{2}\left(1-e^{-u}\right) T(t), \\
\frac{d N(t)}{d t} & =r_{3} N(t)\left(1-\beta_{2} N(t)\right)-c_{2} T(t) N(t)-a_{3}\left(1-e^{-u}\right) N(t), \\
\frac{d u(t)}{d t} & =v(t)-d_{1} u(t) .
\end{aligned}
$$

Here, $N(t)$ is the normal or host cells population, and $u$ is the amount of chemotherapy at the tumour site in the host over time. For $\tau=0$, the model is reduced to ODEs model developed by de Pillis and Radunskaya in [24]. In addition, the reaction of immune cells and tumour cells can result in either the death of tumour cells or the inactivation of the immune cells, resulting in the two competition terms $-\mu E(t-\tau) T(t-\tau)$ and $-n E T$. The tumour cells and normal cells are modelled by a logistic growth law, with parameters $r_{i}$ representing the growth rate of two types of cells: $i=2$ identifies the parameter associated with tumour, and $i=3$ identifies the one associated with the normal tissue, $\beta$ and $\beta_{2}$ are the reciprocal carrying capacities of tumour cells and host cells respectively. In addition, there are two terms representing the competition between tumour and host cells $-c_{1} N T$ and $-c_{2} N T$.

We assume that the drug kills all types of cells, but that the killing rate differs for each type of cells, $a_{i}\left(1-e^{-u}\right)$ is the fraction cell kill for a given amount of drug, $u$, at the tumour site. We denote by $a_{1}, a_{2}$, and $a_{3}$ the three different response coefficients. $v(t)$ represents the amount of dose that is injected into the system, while $d_{1}$ is the decay rate of the drug once it is injected. In this case, the quantity we will control directly is not $u$, but $v(t)$.

It is easy to show that there exists a unique solution of system (4) with initial data $\left(E_{0}, T_{0}, N_{0}, u_{0}\right) \in C$. For biological reasons, we assume that the initial data for system (4) satisfy

$$
E_{0} \geq 0, \quad T_{0} \geq 0 \quad N_{0} \geq 0, \quad u_{0} \geq 0 .
$$

Optimality in treatment might be defined in a variety of ways. Some studies have been done in which the total amount of drug administered is minimized, or the number of tumour cells is minimized. The general goal is to keep the patient healthy while killing the tumour. 
Since our model takes into account the toxicity of the drug to all types of cells, our control problem consists of determining the function $v(t)$ that will maximize the amount of effector cells and minimize the number of tumour cells and the cost of the control with the constraint that we do not kill too many normal cells. If the units of cells are normalized, so that the carrying capacity of normal cells is 1 , we then require that the number of normal cells stay above three-fourth of the carrying capacity. Therefore, in the language of optimal control theory, we should provide an objective functional

$$
\mathcal{J}(v)=\int_{0}^{t_{f}}\left(E-T-\frac{B_{v}}{2}[v(t)]^{2}\right) d t
$$

where $B_{v}$ is a weight factor that describes the patient's acceptance level of chemotherapy; and a constraint

$$
k\left(E, T, N, u, E_{\tau}, T_{\tau}, v\right)=N-0.75 \geq 0, \quad 0 \leq t \leq t_{f} .
$$

We choose the control parameter as a class of piecewise continuous functions defined for all $t$ such that $0 \leq v(t) \leq v_{\max }<\infty$, where $v(t)=v_{\max }$ represents the maximum chemotherapy, while $v(t)=0$ represents the case where there is no chemotherapy. Thus, we depict the class of admissible controls as

$$
V=\left\{v(t), \text { piecewise continuous, } \mid 0 \leq v(t) \leq v_{\max }<\infty, \forall t \in\left[0, t_{f}\right]\right\} .
$$

If we consider the objective functional as a function of $v$, it can be seen that $J$ is concave. Hence, a maximum value can be obtained. The goal is to characterize the optimal control $v^{*}$ satisfies

$$
\max _{0 \leq v \leq v_{\max }} \mathcal{J}(v)=\mathcal{J}\left(v^{*}\right)
$$

\subsection{Existence of an optimal control}

To prove the existence of the optimal control for the underlying model (4), we use the results of Fleming and Rishel [25, Theorem 4.1, p. 68-69]

Theorem 1 There exists an optimal control $v^{*} \in V$ for system (4) such that

$$
\mathcal{J}\left(v^{*}\right)=\max _{v \in V} \mathcal{J}(v)
$$

if the following conditions are satisfied: (i) The set of controls and corresponding state variables is nonempty; (ii) The control set $V$ is convex and closed; (iii) The right-hand side of the state system is bounded by a linear combination of the state and control variables; (vi)The integrand of the objective functional is concave on $V ;(v)$ There exist constants $h_{2}$, $h_{3}>0$ and $b>1$ such that the integrand $L(E, T, v)$ of the objective functional satisfied $L(E, T, v) \leq h_{2}-h_{3}(|v|)^{b}$.

Proof. In order to verify the above conditions, we should first prove the existence of the solution for system (4). Since $\frac{\rho T(t-\tau)}{\eta+T(t-\tau)}<\rho, v_{\max }<\infty$ and by neglecting the negative terms in the model, we have

$$
\frac{d E}{d t}<\sigma+\rho E(t-\tau), \quad \frac{d T}{d t}<r_{2} T, \quad \frac{d N}{d t}<r_{3} N, \quad \frac{d u}{d t}<v_{\max } .
$$

System (9) can be rewritten in the form:

$$
\left(\begin{array}{l}
E \\
T \\
N \\
u
\end{array}\right)^{\prime}<\left(\begin{array}{cccc}
0 & 0 & 0 & 0 \\
0 & r_{2} & 0 & 0 \\
0 & 0 & r_{3} & 0 \\
0 & 0 & 0 & 0
\end{array}\right)\left(\begin{array}{c}
E(t) \\
T(t) \\
N(t) \\
u(t)
\end{array}\right)+\left(\begin{array}{cccc}
\rho & 0 & 0 & 0 \\
0 & 0 & 0 & 0 \\
0 & 0 & 0 & 0 \\
0 & 0 & 0 & 0
\end{array}\right)\left(\begin{array}{c}
E(t-\tau) \\
T(t-\tau) \\
N(t-\tau) \\
u(t-\tau)
\end{array}\right)+\left(\begin{array}{c}
\sigma \\
0 \\
0 \\
v_{\max }
\end{array}\right)
$$


where $^{\prime}=d / d t$. This system is linear in finite time with bounded coefficients. Then the solutions of this linear system are uniformly bounded. Therefore, the solution of the nonlinear system (4) is bounded and exists [26]. Hence, condition one is satisfied.

Clearly, the second condition is satisfied by the definition of $V$. System (4) is bilinear in the control variable $v$ and can be rewritten as

$$
\vec{F}(t, \vec{X}(t), \vec{X}(t-\tau), v)=\vec{\alpha}(t, \vec{X})+\vec{\beta}(t, \vec{X}(t-\tau))+\sigma+v
$$

where $\vec{X}(t)=(E, T, N, u), \vec{X}(t-\tau)=(E(t-\tau), T(t-\tau), N(t-\tau), u(t-\tau)), \vec{\alpha}$ and $\vec{\beta}$ are the vector valued functions of $\vec{X}(t)$ and $\vec{X}(t-\tau)$ respectively. Using that the solutions are bounded, we have

$$
|\vec{F}(t, \vec{X}(t), \vec{X}(t-\tau), v)| \leq\left|F_{1} X(t)\right|+\left|F_{2} X(t-\tau)\right|+\left|F_{3}\right|+\left|F_{4}\right| \leq h_{1}|\vec{X}|+|\sigma|+|v|
$$

where $h_{1}$ depends on the coefficients of the system, and

$$
F_{1}=\left(\begin{array}{cccc}
0 & 0 & 0 & 0 \\
0 & r_{2} & 0 & 0 \\
0 & 0 & r_{3} & 0 \\
0 & 0 & 0 & 0
\end{array}\right), F_{2}=\left(\begin{array}{cccc}
\rho & 0 & 0 & 0 \\
0 & 0 & 0 & 0 \\
0 & 0 & 0 & 0 \\
0 & 0 & 0 & 0
\end{array}\right), F_{3}=\left(\begin{array}{c}
\sigma \\
0 \\
0 \\
0
\end{array}\right), F_{4}=\left(\begin{array}{l}
0 \\
0 \\
0 \\
v
\end{array}\right) .
$$

We also note that the integrand of $J(v)$ is concave in $V$. Finally,

$$
E-T-B_{v} / 2[v(t)]^{2}<E-B_{v} / 2[v(t)]^{2} \leq h_{2}-h_{3}|v(t)|^{2},
$$

where $h_{2}$ depends on the upper bounds of $E$ and $T$, and $h_{3}=B_{v} / 2$. This completes the proof.

\subsection{Optimality of system (4)}

We proved in the above section the existence of optimal control system given by the objective functional (5) for system (4). In order to derive the necessary conditions for an optimal control problem, we use Pontryagin's maximum principle with delay [27]. This principle converts (4), (5), and (8) into a problem of minimizing a Hamiltonian functional, $H$ with

$$
H\left(t, E, T, E_{\tau}, T_{\tau}, u, v, \lambda\right)=E-T-\frac{B_{v}}{2}[v(t)]^{2}+\lambda_{1} \frac{d E}{d t}+\lambda_{2} \frac{d T}{d t}+\lambda_{3} \frac{d N}{d t}+\lambda_{4} \frac{d u}{d t}+\gamma k
$$

and $\gamma \geq 0$ with $\gamma(t) k(t)=0$, where

$$
\gamma=\left\{\begin{array}{lc}
1 & \text { if } N(t) \leq 0.75 \\
0 & \text { otherwise }
\end{array}\right.
$$

To minimize the Hamiltonian functional, the Pontryagin's maximum principle [27] is used. Thus, the following theorem is deduced:

Theorem 2 Given an optimal control $v^{*}$ and solutions of the corresponding state system (4), there exist adjoint variables $\lambda_{i}$ for $i=1,2,3,4$ satisfying the following

$$
\begin{aligned}
& \lambda_{1}^{\prime}(t)=-1+\lambda_{1}(t)\left[\delta+a_{1}\left(1-e^{-u^{*}}\right)\right]+\lambda_{2}(t) n T^{*}+\lambda_{1}(t+\tau) \chi_{\left[0, t_{f}-\tau\right]}\left[\mu T^{*}-\frac{\rho T^{*}}{\eta+T^{*}}\right], \\
& \lambda_{2}^{\prime}(t)=1+\lambda_{2}\left[-r_{2}+2 r_{2} \beta T^{*}+n E^{*}+c_{1} N^{*}+a_{2}\left(1-e^{-u^{*}}\right)\right]+\lambda_{3} c_{2} N^{*} \\
& +\chi_{\left[0, t_{f}-\tau\right]} \lambda_{1}(t+\tau)\left[\frac{\rho E^{*} T^{*}}{\left(\eta+T^{*}\right)^{2}}-\frac{\rho E^{*}}{\eta+T^{*}}+\mu E^{*}\right], \\
& \lambda_{3}^{\prime}(t)=\lambda_{2} c_{1} T^{*}-\lambda_{3}\left(r_{3}-2 r_{3} \beta_{2} N^{*}-c_{2} T^{*}-a_{3}\left(1-e^{-u^{*}}\right)\right)-\gamma, \\
& \lambda_{4}^{\prime}(t)=-\lambda_{1}(t) a_{1} e^{-u^{*}} E^{*}+\lambda_{2}(t) a_{2} e^{-u^{*}} T^{*}+\lambda_{3}(t) a_{3} e^{-u^{*}} N^{*}+\lambda_{4}(t) d_{1},
\end{aligned}
$$


with transversality conditions

$$
\lambda_{i}\left(t_{f}\right)=0, \quad i=\{1,2,3,4\} \text { and } \quad \chi_{\left[0, t_{f}-\tau\right]}= \begin{cases}1 & \text { if } t \in\left[0, t_{f}-\tau\right], \\ 0 & \text { otherwise. }\end{cases}
$$

Moreover, the optimal control is

$$
v^{*}=\min \left(v_{\max }, \frac{\lambda_{4}}{B_{v}}\right) .
$$

Proof. The adjoint equations and transversality conditions can be obtained by using Pontryagin's maximum principle with delay in state [27] such that

$$
\begin{array}{ll}
\lambda_{1}^{\prime}(t)=-\frac{\partial H}{\partial E}(t)-\chi_{\left[0, t_{f}-\tau\right]}(t) \frac{\partial H}{\partial E_{\tau}}(t+\tau), & \lambda_{1}\left(t_{f}\right)=0, \\
\lambda_{2}^{\prime}(t)=-\frac{\partial H}{\partial T}(t)-\chi_{\left[0, t_{f}-\tau\right]}(t) \frac{\partial H}{\partial T_{\tau}}(t+\tau), & \lambda_{2}\left(t_{f}\right)=0, \\
\lambda_{3}^{\prime}(t)=-\frac{\partial H}{\partial N}, & \lambda_{3}\left(t_{f}\right)=0 \\
\lambda_{4}^{\prime}(t)=-\frac{\partial H}{\partial u}, & \lambda_{4}\left(t_{f}\right)=0 .
\end{array}
$$

The optimal control $v^{*}$ can be solved from the optimality condition $\frac{\partial H}{\partial v}(t)=0$ that is, $-B_{v} v+$ $\lambda_{4}=0$.

By the bounds in the control set $V$, it is easy to obtain $v^{*}$ in the form of (13). The optimality system consists of the state system (4) coupled with the adjoint system (12) with initial and transverality conditions together with the characterization together with the optimal control. Therefore, the optimal system is given as follows:

$$
\begin{aligned}
\frac{d E^{*}}{d t} & =\sigma+\frac{\rho E^{*}(t-\tau) T^{*}(t-\tau)}{\eta+T^{*}(t-\tau)}-\mu E^{*}(t-\tau) T^{*}(t-\tau)-\delta E^{*}-a_{1}\left(1-e^{-u^{*}}\right) E^{*}, \\
\frac{d T^{*}}{d t} & =r_{2} T^{*}\left(1-\beta T^{*}\right)-\mu E^{*}(t) T^{*}-c_{1} N^{*} T^{*}-a_{2}\left(1-e^{-u^{*}}\right) T^{*}, \\
\frac{d N^{*}}{d t} & =r_{3} N^{*}\left(1-\beta_{2} N^{*}\right)-c_{2} T^{*} N^{*}-a_{3}\left(1-e^{-u^{*}}\right) N^{*}, \\
\frac{d u^{*}}{d t} & =v^{*}-d_{1} u^{*}, \\
\lambda_{1}^{\prime}(t) & =-1+\lambda_{1}(t)\left[\delta+a_{1}\left(1-e^{-u^{*}}\right)\right]+\lambda_{2}(t) n T^{*}+\lambda_{1}(t+\tau) \chi_{\left[0, t_{f}-\tau\right]}\left[\mu T^{*}-\frac{\rho T^{*}}{\eta+T_{f}}\right] \\
\lambda_{2}^{\prime}(t) & =1+\lambda_{2}\left[-r_{2}+2 r_{2} \beta T^{*}+n E^{*}+c_{1} N^{*}+a_{2}\left(1-e^{-u^{*}}\right)\right]+\lambda_{3} c_{2} N^{*} \\
\lambda_{3}^{\prime}(t) & =\lambda_{2} c_{1} T^{*}-\lambda_{3}\left(r_{3}-2 r_{3} \beta_{2} N^{*}-c_{2} T^{*}-a_{3}\left(1-e^{-u^{*}}\right)\right)-\gamma \\
\lambda_{4}^{\prime}(t) & =-\lambda_{1}(t) a_{1} e^{-u^{*}} E^{*}+\lambda_{2}(t) a_{2} e^{-u^{*}} T^{*}+\lambda_{3}(t) a_{3} e^{-u^{*}} N^{*}+\lambda_{4}(t) d_{1}, \\
v^{*} & =\min \left(v_{\max }, \frac{\lambda_{4}}{B_{v}}\right) \\
E^{*}(\theta) & =\psi_{1}(\theta), T^{*}(\theta)=\psi_{2}(\theta), N^{*}(\theta)=\psi_{3}(\theta), u(\theta)=\psi_{4}(\theta), \theta \in[-\tau, 0], \\
\lambda_{i}\left(t_{f}\right) & =0, i=\{1,2,3,4\} .
\end{aligned}
$$


The numerical approximations of the optimal control problem are carried out using forward and backward Euler methods. Starting with an initial guess for the value of the controls on the time interval $\left[0, t_{f}\right]$, we solve the state system with control variables (4) using forward Euler' scheme. While, the adjoint system is solved using the solutions of the state system and the transversality conditions backward in time. The Pontryagin-type maximum principle has been derived for retarded OCP, with delays in the state variable when the control system is. subject to a mixed control state, in order to minimize the cost of treatment, reduce the tumour cells load, and keep the number of normal cells above $75 \%$ of its carrying capacity.

Figure 1 shows the impact of chemotherapy treatments (with optimal control) when we choose the parameter values in an unstable region $(\sigma=0.2, \rho=0.2$, and $\tau=1.5)$. The tumour cell population is growing up over time in the absence of chemotherapy, while the presence of treatment helps the immune system to keep the growth of the tumour cells under its control. The Figure shows that the tumour cells can be eradicated at day 20, which in some degree meets the natural facts.
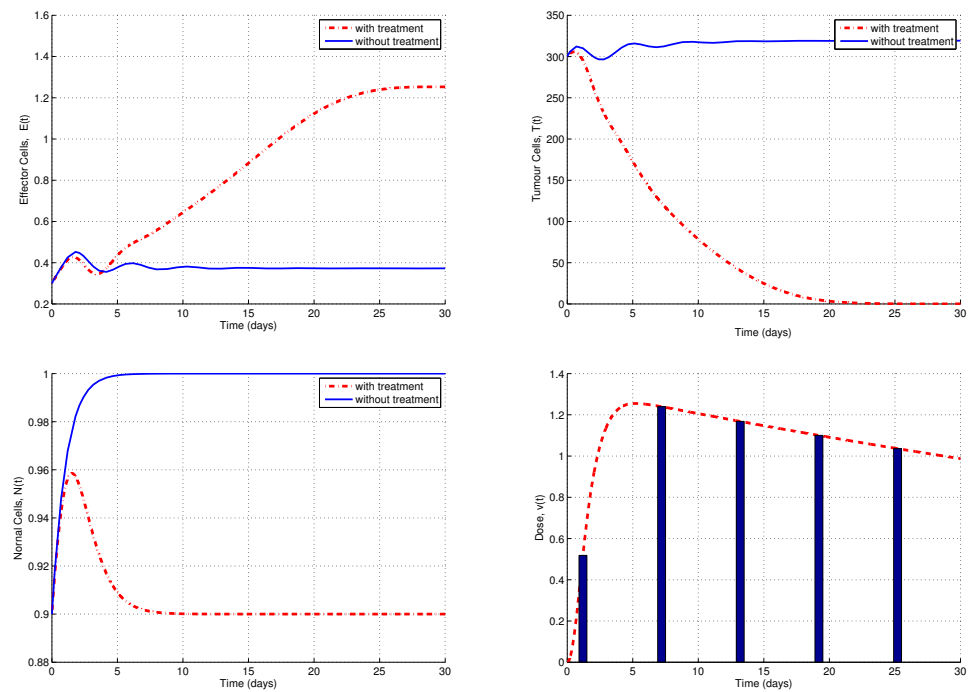

Figure 1. Simulations of system (15), in the stable region, before and after the treatments with control, initial conditions $E_{0}=0.3, T_{0}=300$, and $N_{0}=0.9$ and the parameter values are given in the text. The load of tumour cells is growing up over time in absence of treatment; While presence of chemotherapy helps the immune system to keep the growth of the tumour cells under its control.

\section{Discussion and Conclusion}

Herein, we provided a mathematical model with time-lags and optimal control variables to describe the dynamics of tumour-immune interactions in presence of chemotherapy treatments. The numerical approximations of the optimal control problem are carried out using forward and backward Euler methods. Starting with an initial guess for the value of the controls on the time interval $\left[0, t_{f}\right]$, we solve the state system with control variables (4) using forward Euler' scheme. While, the adjoint system is solved using the solutions of the state system and the transversality conditions (12) backward in time. The Pontryagin-type maximum principle is derived, for retarded optimal control problems with delays in the state variable when the control system is subject to constraints. This is to minimize the cost of treatment, reduce the tumour cells load, and keep the number of normal cells above $75 \%$ of its carrying capacity. 
The numerical simulations validate the existence of optimality of the control variables. The presence of chemotherapy protocol reduces the tumour load in few days of therapy. Of course, this model can be extended to more powerful models for cancer treatment design with optimal combinations, doses, and scheduling of treatments to speed up the development of individualized therapies.

\section{References}

[1] M. Martins, S.C.J. Ferreira, M. Vilela, Phys. Life Rev. 4, 128 (2007)

[2] R. Araujo, D. McElwain, Bull. Math. Biol. 66, 1039 (2004)

[3] N. Bellomo, N. Li, P. Maini, Math. Mod. Methods Appl. Sci. 18, 593 (2008)

[4] N. Bellomo, L. Preziosi, Math. Comput. Model 32, 413 (2000)

[5] H. Byrne, T. Alarcon, M. Owen, S. Webb, P. Maini, Philos. Trans. R. Soc. A 364, 1563 (2006)

[6] F. Castiglione, B. Piccoli, J. Theor. Biol. 247, 723 (2007)

[7] V. Kuznetsov, I.A. Makalkin, M. Taylor, A. Perelson, Bull. Math. Biol. 56, 295 (1994)

[8] S. Banerjee, Int. J. Appl. Math. Comput. Sci. 18, 389 (2008)

[9] R. Eftimie, Bull. Math. Biol. 73, 2 (2011)

[10] F.A. Rihan, S. Muntaser, M.A. Abdeen, D.H. Abdelrahman, J. Appl. Math. 2012, 19 (2012)

[11] N. Buric, M. Mudrinic, N. Vasovic, Chaos, Solitons and Fractals 12, 483 (2001)

[12] R. Yafia, Nonlinear Anal. Model. Contr. 11, 95 (2006)

[13] F.A. Rihan, D.H. Abdelrahman, Int. J. Comput. Math. 90, 594 (2013)

[14] U. Forys, M. Mochar, J. Polezzczuk, Appl. Math. Letters p. 982986 (2011)

[15] F.A. Rihan, S. Lakshmanan, H. Maurer, Applied Mathematics and Computation 353, 147 (2019)

[16] H.T. Banks, Modelling and control in biosciences, Vol. 6 (Lect. Notes in Biomath: Springer, Berlin, 1975)

[17] V.B. Kolmanovskii, L. Shaikhet, Control of systems with aftereffect (American Mathematical Society, 1996)

[18] S.E. Smith, Complexity International 12, 1 (2005)

[19] F.A. Rihan, B.F. Rihan, Appl. Math. \& Inf. Sci. 9, 1615 (2015)

[20] Optimal Control Applied to Biological Models (Chapman and Hall/CRC, New York, 2007)

[21] K. Hattaf, N. Yousfi, ISRN Biomathematics 2012, 7 pages (2012)

[22] A. Rundell, R. DeCarlo, V. Balakrishnan, H. HogenEsch, IEEE. Trans. Biomed. Eng. 45, 429 (1998)

[23] F.A. Rihan, D. Abdelrahman, S. Lakshmanan, A. Alkhajah, Appl. Math.Compu. 232, 606 (2014)

[24] L.G. de Pillis, A. Radunskaya, Comput. Math. Methods Med. 3, 78 (2001)

[25] W.H. Fleming, R.W. Rishel, Deterministic and Stochasitic Optimal Control (SpringerVerlag, New York, 1994)

[26] D.L. Lukes, Differential Equations: Classical to Controlled (Academic Press, New York, 1982)

[27] L.S. Pontryagin, R.V. Boltyanski, R.V. Gamkrelidge, E.F. Mischenko, The Mathematical Theory of Optimal Processes (John Wiley and Sons, N.Y., 1962) 